\title{
Human Identification in Smart Home Environment
}

\author{
S. Shakthi Priyadharshini, A. Umamakeswari
}

\begin{abstract}
People moved their life as automatic systems, like smart home. But here major issues is security, the sensitive personal information have been raised the privacy concerns such as (modifying the data, unauthorized access of data) among peoples would like to share their personal information are only utilized for their benefits, rather than being utilized for malignant purpose. Person Identification in smart home with real time is the major challenging in monitoring system. Capturing the still and live images with high efficiency is the complex task. This system provides the efficiency in higher rate with quick response. Data are lively gathered and lively compared with the data, so the process is undergoing the HAAR and the Convolutional Neural Network (CNN). Capturing and labeling the images is done with the live processing then the live video processing is taken the frames of images. So, the data are not stored for long time, and compared the still and live frames in the known frame databases it matches the features and notify the system with two categories as known and unknown.
\end{abstract}

Index Terms: Human Classification, Face Recognition, HAAR Cascade, CNN, Person Re-Identification

\section{INTRODUCTION}

Internet of things (IOT) reduces the manpower in every field. Internet of things developed in now-a-days but there is an increasing challenge such as protecting the hardware and prevent data from malicious attacks and unauthorized access. Here human identity is taken as data and the uniqueness if the data monitoring is become more difficult.This primarily concentrations on the numerous approaches that develop the smart home model.In this article human identification is not recognized using wearable tags instead rest on unique signature is taken from every individuals like IR rays, heartbeat and respiration system of human.CNN is an efficient method for object recognition using deep learning[1].In this scenario each movement of the person is monitored by using capacitance and that capacitance is operates on load manner. From this approach every human has unique signature, so it differs mass index for every person as well as it produces different frequencies. So those frequencies are calculated by RC low pass. The main advantage of this system is low cost as well as low power [2]. This approach mainly concentrates on the privacy and security. In this system human actions are monitored.

Revised Version Manuscript Received on Jun 20, 2019.

S.Shakthi Priyadharshini. School of Computing, SASTRA Deemed

University, Thanjavur, Tamil Nadu-613401

A.Umamakeswari. School of Computing, SASTRA Deemed University, Thanjavur, Tamil Nadu-613401.
The simultaneous events are going-on like playing games even though talking with an individual are measured. Including these actions are also considered like cooking and cutting of vegetables. Here there is some break in the human action, and they do not do both the actions at the same time. Human existence is noticeable by symbols and analyzed by using JAVA platform. During some time, interval of human action, the filtration process is performed here improves the performance and privacy concern [3]. In UWB radar approach, human breath is monitored by using time domain reflectometry. UWB have the capability to observe the patient breath without having physical interaction. The major disadvantage of the system is limited number of masks only provided and taking many computing resources and it is very difficult operation [4]. In this paper, the walking behavior of the person is analyzed and the sounds has been recorded which are stored in the database system. By applying these techniques that is Short-Time Fourier Transform (STFT) and Teager-Kaiser Energy Operator (TKEO) it evolves energy frequencies as well as amplitude of footsteps. Teager-Kaiser Energy Operator separates the different footstep sounds from the different ground surfaces [5]. In this report the Blue Sound sensors have the capability to identifying the individual person who exists in the house. The Passive Infrared (PIR) sensor is used to identify the human pattern with less positive rate so the plenty of PIR sensors is fixed to monitor the human actions. When human crosses the sensor zone, at that time every resident height is calculated by Ultrasound sensor. Disadvantage of the system is to give lot of burden [6]. This paper focuses on forest areas and to find the how many numbers of persons inside in the forest and it is calculating by hybrid wavelet independent factor. Then sending and receiving signals purpose use the Time domain dipole antennas and Impulse radio. But the major drawback is to limits in target identification and the Underprivileged visibility circumstances [7]. method is used for joint structures and skin tone of the person and it's to find parts of human body. Well thought-out maps are major role in tracking of human actions. This system efficiently focuses in the outline structure of human body from the loud circumstantial [8]. To excerpt the individual signature by Region of Interest (ROI) and then human form is categorized by Principle Component Analysis (PCA) and Support Vector Machine (SVM). Labelling the input images is the more time-consuming process. A general way is to label the small subset of images is to identify, the originality of outcome. Initially training the model (e.g., a classifier) based up on the labelled data, which can be further used to identify the 
importance of the unlabelled data (i.e., unknown). After the outcome of dataset validation, generate the ranking criteria for all unlabelled samples based on the label allocation. The given information for every small subset of images could bring the more detailed descriptions for understanding every visual content in their repository or real time scenario.

We can get more benefit from the additional text information associated with web images during the learning procedure, can help to find accurately the origin of images. Recognition of the human can be classified with different parameters and designs it could be differing in only the area like eyes and mouth detections. Similarity identification and rejection is more complex task. Cascade for person face and classifier for eyes, nose, and mouth recognitions are consider as a weak classifier then the entire body classification can be used for method as stronger. Plane or axis is useful to identify the parts of body and combine the several overlying identifications to section for single identity of human. In Received Signal Strength (RSS) foot printing method there are two major features one is an acquisition scheme and another one is a server [9]. Radio frequency signal faces various issues such as locating the position owing to signal transmission. Using the Bluetooth scanner RSS send and receive the information. And this information is directed to central organization via Wi-Fi TCP sockets. This process calculates the sensor positions and further analyzing with the help of cloud platform. By getting the least error function at the time of positions is evaluated by matching with already trained systems. Ultra-wideband sensor is a wireless technology for identifying or monitoring the human and transmitting the data, with high speed and short range, but it consumes the low transmitting energy and hard to intercept. UWB is not only for general human identification it can also be applied for the medical moment tracking like breath moment monitoring. Another method for indoor localization is Bluetooth low energy technology [10]. This system needs the data aggregation, sensing and transmitting power but it consumes low power. This collects all the data are grouped in SQL and it is transmitted throughout the UNIX sockets. Database predicts the position of the nodes. Major drawback of this system is location estimation becomes more difficult and incorrect process. Multiple Input and Multiple Output (MIMO) is another technique which is used to analyze the heartbeat and human respiration [11]. The collected data are utilized for the further evaluation function. After the evaluation function the correlation reaches the threshold. DC will reduce the recognition functionality. The channel is calculated in the discrete time points. In order to eliminate the DC time averaged channel is deducted from the observed channel. Person identification can be done using the microcontroller when capacitance is used to collect the sensor signals and resistance is used to demodulate the signals using XOR gate. Here microcontrollers are used to generate the sine signals and long capacitive sensor is used to achieve the higher accuracy in recognition. To reduce the tagging concept that is long range capacitive sensor. Effectively reduces the noise that is occurred by the sensor transducer. To identifying the person multiple times frequency is passed on the person [17]. MAS render services based on RFID technology. As RFID is less compatible with the wireless technologies, NFC protocol promotes efficient identification of human. It utilizes electromagnetic field to communicate with the other devices. In addition to this, the patient's body is attached with body area network (BAN) which extracts the patient's state of health. This system not only gathers information about the residents but also analyses the information to detect the anomalies. Belief, desire and intention are the major factors which are computed for patient's treatment. A catcher agent is used to accumulate all the information and send it to the head agent. All the data collected are organized by the data agent. The head agent in turn informs the application agent in accordance to the priority of the incidence. The two important phases implemented are case based reasoning and case-based planning. The reasoning mechanism in this module creates the plan to take care of the patients based on previous experiences [18]. To identifying the human body pyro electric sensor is used it increases the efficiency and reduced the false positive rate. Various sensor types can be merged to cover the various height rates. Tracking the human using different methods like RFID tag but it makes people to feel more complex and there is a chance to make any fraudulence and if we cross the area then automatically it gathers the id information and counted as present information. The RFID tag and IR transmitter is used to recognize the human in localization only and the collected information is transferred using the RBF network. But it achieves the error distance rate as 2.5 meters. This method gives an extra benefit of adding and eliminating of the sensors simply without any sort of tedious efforts. A Seamless (environment intelligent) and Seamful (recognizing the person with sensors) sensor decreases the essential of several sensors. The key concept of this method is creating the surrounding more powerful. Combined raised area acts as a bond between these sensors for give-and-take of messages [20]. A hierarchical layer is to improve the consequence with divide and conquer system. Advantages of the Seamful sensors is good to consuming the power and efficiently deal with unpredicted ambiguities.

\section{HUMAN IDENTIFICATION USING CLASSIFICATION MECHANISMS}

Human detection is carried out by various technologies such as face recognition, Fingerprint recognition, Eye pupil recognition etc.; Generally, face recognition is carried out by many parameters like angles and region of nose, eyes. All detection technique is underlying with some classification or detection algorithms. Here we perform the detection and classification scheme with two technologies such as HAAR and CNN. To increase the accurateness of face gratitude in videos to get robust and scalable identification can be attained by the material of frames and features of poses, in video frame the collected features of data is combined into the set of data for recognition.

\section{A. Haar:}

HAAR is used to detect the object. Here it is detecting the object as lively and stores in the database then it is lively compared with the frames. Once the person is stored with the label it is carried over for the full process if any new data are 
appeared in the dataset then it is counted as a new data for further process or it is detected as an unknown data.

Images are captured in the still moment and saved for future process and its copy is taken as input for dataset. Moving images such as videos are taken in the format of frames each frame is encounter the different angle of face gestures. Input is not directly taken as images it is transformed in to the matrix. This representation states the original data of image.

Frame of images is stored in the same matrix format; it is used for the comparison.

\section{B. HAAR Cascade Functionality:}

HAAR cascade is a feature like adjacent rectangular region within the bounding box it selects the human facial pose. Different pixel values are calculated in the different bounding box and the whole value is summing up together to find out the single sequence of image.

After the capturing of image, it validates the features with the input parameter such as (eyes, nose, and mouth) as well as covered the face angle.

\begin{tabular}{|l|l|l|l|}
\hline 0 & 8 & 6 & 1 \\
\hline 1 & 5 & 9 & 0 \\
\hline 0 & 7 & 5 & 0 \\
\hline 2 & 8 & 9 & 2 \\
\hline
\end{tabular}$\quad$\begin{tabular}{|l|l|l|l|}
\hline 0 & 8 & 14 & 15 \\
\hline 1 & 14 & 29 & 30 \\
\hline 1 & 21 & 41 & 42 \\
\hline 3 & 31 & 60 & 63 \\
\hline
\end{tabular}

Image

Integral Image

\section{Fig.1 Integral Image}

HAAR Cascade needs a set of input datum and a set of real time entry images. A set of detected data with regions of interest is used to predict the original data. The Image Labeler to be used for labelling the objects of interest with the bounding boxes.

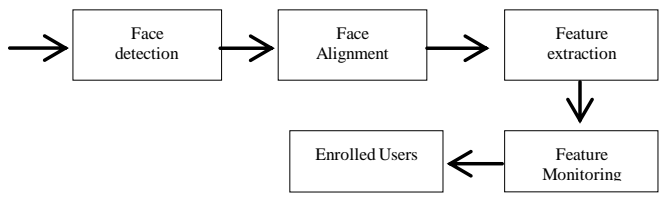

Fig2. Process of HAAR

\section{A. CNN:}

A Convolutional Neural Network is a feed-forward network which extracts the property of input images. Extracts the features from the raw images further it classifies the features of images. In input layer it accepts input as pixel of arrays further it is applied on hidden layer and extract the feature of images. Dataset image is converted on to the $5 \times 5$ matrix format then the real time images are converted into the co-variance matrix. Then it is matched with the original matrix and the co-variance matrix if it matches then conclude the known person if the matrix is not match then it classifies the unknown person. This feature reduces the number of trained parameter because the dataset is taken from the real time environment. Images are in the representation format such as $\mathrm{X}$ and $\mathrm{Y}$ axis. The images are first captured and saved with the name of source and details then image is lively focused the frame is detected if any unknown then it notifies in statement. Multiple feature maps are applied on the multiple images. Images are taken as the sub samples and check the facial features it reduces the resolution of feature map, reduces the false negative rate. Images are transformed in to scaling property it helps us to increase the feature selection as efficient.

$\mathrm{CNN}$ is used for classifying the detected image. Simply it is stated as known or unknown. In other word it is stated as decision making process. Scenario of detection and classification is explained as input and validation format. In every offset of $\mathrm{x}$ or $\mathrm{y}$ coordinates is placed on the corner of left top or the right bottom which is reverted from convolution layer.

\section{PROPOSED WORKING FLOW}

Steps to Detect and Classification:

Step 1: Registering the new user

Step 2: Labelling the new data with unique identity

Step 3: Collecting the features of data with the detailed information of person

Step 4: Collected datum are stored in database for further classification process

Step 5: Live video capturing with number of frames

Step 6: Frames are transformed in axis format and changed the pattern as gray scale property

Step 7: Identify the features of face

Step 8: Classify the images with label and identify the similarity with the detected

Still image phase
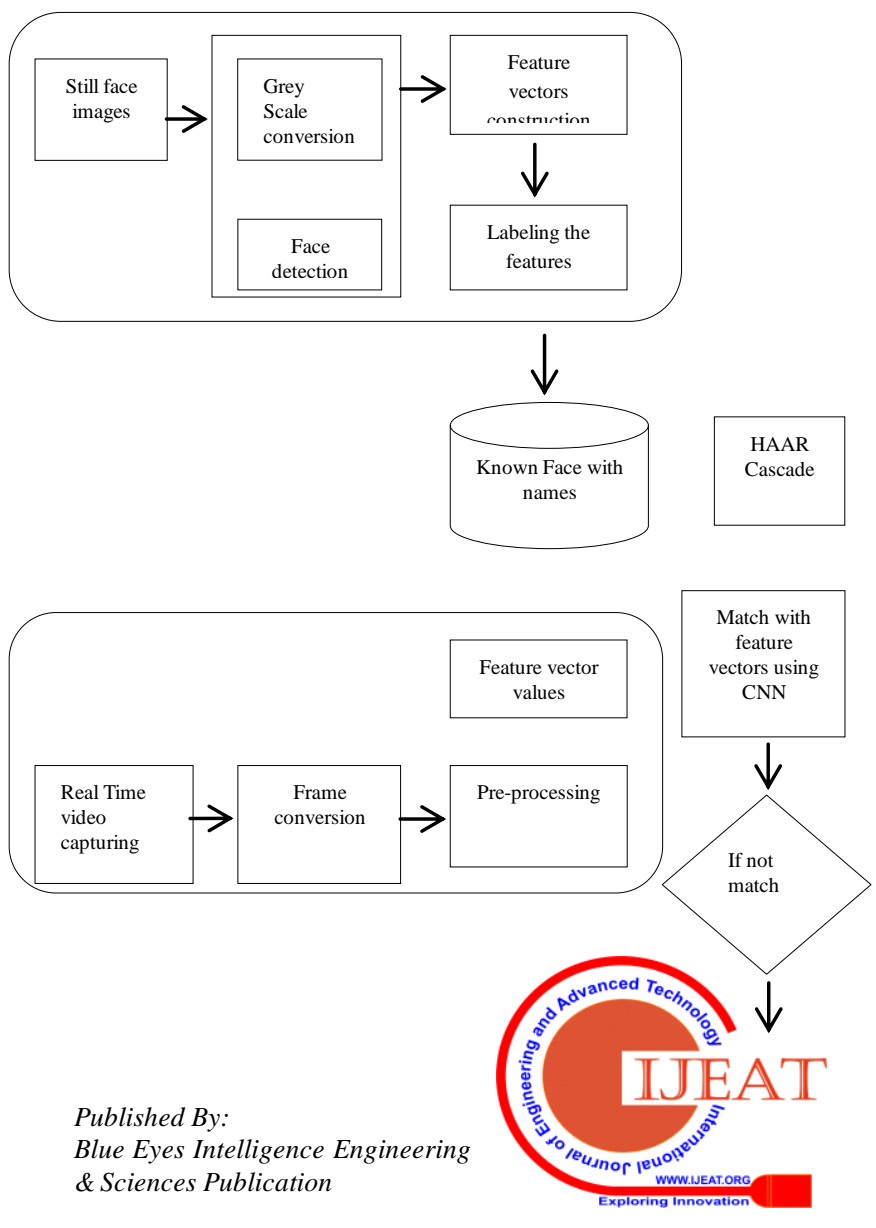


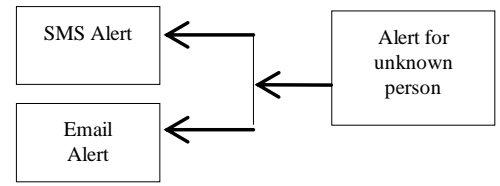

Fig3. Proposed flowchart for human identification

In proposed system, there are two phases one is a still image phase and the other one is a real time video phase. From that still image phase process, the person captures his face and then processed image are convert to a matrix of axis data, using the grayscale color map representation, the intensity " 0 " is represents as black and the intensity " 1 " is represents as white.

Image recognition systems must perform the grayscale images, with the converting mechanism of color-to-grayscale. After the conversion of normal images to grey scale the face angles are detected and extract the feature. Extracting the face feature plays major role in identification. Feature extraction can vary for different parameter (e.g. eyes, nose, and mouth).Feature extraction is a process of dimensionality reduction, where a set of raw variables is reduced to sub sets of groups (features) for processing, and the better feature extracted data is used for further reference (i.e., Database).

Another one is a real time video phase, during live streaming the moving images are counted as single frames; first detect the face using bounding box and then identifying the face position after that image is cropped. Similarly, for live streaming process face feature extraction is used for identifying the human and then decision-making process is carried out by CNN. Here it is identifying that whether the person is already in database or not if it is in database then it states that person is known if it is not in database or it is not identify the person with existing data then it states that the person is unknown.

Matching process is carried by the matrix format the original data is stored in $5 * 5$ and the lively captured images are also formed as covariance matrix. The decision is made by the comparison of original matrix with the transpose of covariance matrix if these two matrices are same then it is matched with the image else it is denoted as unknown.

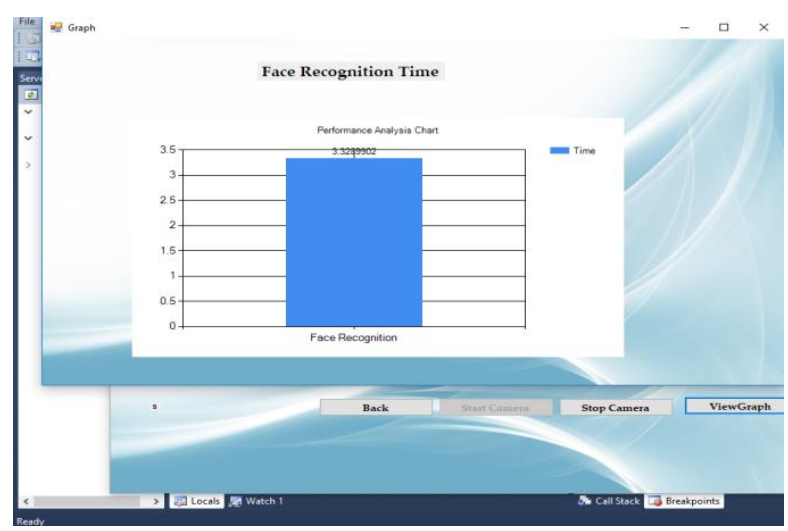

Fig4. Performance Analysis graph for the real-time live streaming

\section{Result Analysis}

In above graph is represented the performance analysis. It is the analysis; it differs for every different input. Calculation is done with the recognition time. The time from the image is captured and time taken to recognize the feature is the parameter to calculate the efficiency. It is very efficient because it consumes only minimum amount of time to recognize the feature when it is compared to SVM and PCA. The average area covering is based on the camera what we used, and the resolution of the images are varying for every different camera. Angular trajectory can also be achieved if we are using the full angle coverage camera, other parameters like resolution and quality is based on the camera and not rely on the algorithm we used. Thus, the analysis is estimated in timely manner and it achieves minimum time consumption. Further the project can be extended by other some machine learning algorithms like (Logistic regression, Random forest).

\section{CONCLUSION}

In this paper, we performed face recognition technique for still and moving images (i.e.,) videos. Most of the approaches focus only the still images and video-to-video images. This is not efficient for the current scenario because it limits the number of input images extracted from the videos; there is a constraint in the resources for calculating the real time processing. Future, it will be proceeding with the still-to-still, still-to-video and video-to-video matching in real-time. HAAR Cascade is gives the efficient outcome when compared to SVM and PCA in terms of time taken. This method is executed by the real time processing and data set is taken from the real time implementation not in the form of test and trained form. Alert system is used to notify the unknown person if the system find the known person then it simply valuates, if it find out any controversy it check the alert value, it is fixed by the Threshold value such as 5 if it gives less than the threshold then it send the alert notification to the corresponding user. A result shows that higher recognition rates are obtained by using the efficient detection and classification technique.

\section{REFERENCES}

1. Shih-Chung Hsu and Yu-Wen Wang, Chung-Lin Huang" Human Object Identification for Human-Robot Interaction by using Fast R-CNN 2018 Second IEEE International Conference on Robotic Computing-2018.

2. Javed Iqbal, Mihai TeodorLazarescu, Osama Bin Tariq, "Capacitive Sensor for Tag less Remote Human Identification Using Body Frequency Absorption Signatures," IEEE Transactions On Instrumentation And Measurement, Volume: 67, Issue: 4, April 2018.

3. RaduProdan, IoanNascu, "Identifying Patterns for Human Activities of Daily Living in Smart Homes," IEEE International Conference on Automation, Quality and Testing, Robotics, 2014.

4. S. Pisa, E. Pittella, E. Piuzzi, M. Cavagnaro, and P. Bernardi., "Design of a UWB Radar System for Remote Breath Activity Monitoring" IEEE/MTT-S International Microwave Symposium Digest,2012.

5. M. Umair Bin Altaf*, TarasButko, and Biing-Hwang (Fred) Juang, "Acoustic Gaits: Gait Analysis with Footstep Sounds," IEEE Transactions on Biomedical Engineering, Vol. 62, NO. 8, AUGUST 2015 .

6. G. Mokhtari,Q. Zhang,S. Ball, M. Karunanithi "BLUESOUND: A New Resident Identification Sensor - Using Ultrasound Array and BLE Technology for Smart Home Platform," DOI 10.1109/JSEN.2017.2647960, IEEE Sensors Journal.

7. Xiangbo He, Ting Jiang, "Target identification in foliage environment 
using UWB radar with hybrid wavelet-ICA and SVM method," Journal Physical Communication, Feb 2014.

8. Adnan Farooq, Ahmad Jalal, and Shaharyar Kamal, "Dense RGB-D Map-Based Human Tracking and Activity Recognition using Skin Joints Features and Self-Organizing Map," KSII TRANSACTIONS ON INTERNET AND INFORMATION SYSTEMS VOL. 9, NO. 5, May 2015.

9. Marco Teran, Juan Aranda and Henry Carrillo, Diego Mendez and Carlos Parra "IoT-based System for Indoor Location using Bluetooth Low Energy, count, location, track, and identity," IEEE Colombian Conference on Communications and Computing (COLCOM), 2017.

10. S. Pisa, E. Pittella, E. Piuzzi, M. Cavagnaro, and P. Bernardi., "Design of a UWB Radar System for Remote Breath Activity Monitoring" IEEE/MTT-S International Microwave Symposium Digest,2012.

11. Dai Sasakawa, Naoki Honma, Takeshi Nakayama, "Human Identification Using MIMO Array", IEEE Sensors Journal, Volume: 18, Issue: 8, April15, 152018

12. VICTORIA MORENO ; MIGUEL A. ZAMORA ; ANTONIO F. SKARMETA, A LOW-COST INDOOR LOCALIZATION SYSTEM FOR ENERGY SUSTAINABILITY IN SMART BUILDINGS IEEE SENSORS JOURNAL, 2016.

13. IBRAHIM AL-NAIMI ; CHI BIU WONG ; PHILIP MOORE ; XI CHEN, "INDOOR IDENTIFICATION AND TRACKING USING ADVANCED MULTIMODAL APPROACH”, 10TH INTERNATIONAL SYMPOSIUM ON MECHATRONICS AND ITS APPLICATIONS (ISMA), 2015.

14. PIREHPIRZADA ; NEIL WHITE, ADRIANA WILDE, "SENSORS IN SMART HOMES FOR INDEPENDENT LIVING OF THE ELDERLY" 5TH INTERNATIONAL MULTI-TOPIC ICT CONFERENCE (IMTIC), 2018

15. Alina Roitberg, Alexander Perzylo, Nikhil Somani, Manuel Giuliani, "Human Activity Recognition in the Context of Industrial Human-Robot Interaction," Signal and Information Processing Association Annual Summit and Conference (APSIPA), 2014.

16. Long Xiao ; Bo Cheng ; Bo Yang ; Rong Du ; Wenbin Yu ; Xinping Guan, "A context-aware entrance guard in smart home: An event-driven application based on the human motion and face recognition" 5th International Conference on Automation, Robotics and Applications, 2011

17. Javed Iqbal, Mihai TeodorLazarescu, ArslanArif and Luciano Lavagno, "High sensitivity, low noise front-end for long range capacitive sensors for tag less indoor human localization," IEEE 3rd International Forum on Research and Technologies for Society and Industry (RTSI), 2017.

18. Ching-Hu Lu, Student Member, IEEE, Chao-Lin Wu, Member, IEEE, and $\mathrm{Li}-\mathrm{Chen} \mathrm{Fu}$, Fellow, IEEE, "A Reciprocal and Extensible Architecture for Multiple-Target Tracking in a Smart Home" IEEE transactions on systems, man, and cybernetics-part c: applications and reviews, Vol. 41, no. 1, January 2011.

19. Frederic Bergeron; Kevin Bouchard; Sylvain Giroux; Sebastien Gaboury ; Bruno Bouchard "Simple objects tracking system for smart homes," IEEE International Conference on Bioinformatics and Biomedicine (BIBM), 2015.

20. Juan A. Fraile, Javier Bajo, Juan M. Corchado, and Ajith Abraham, Senior Member, IEEE, "Applying Wearable Solutions inDependent Environments", IEEE transactions on information technology in biomedicine, vol. 14, no. 6, november 2010.

21. QinyiXu,YanChen,BeiBei Wang: "Radio-Shot Through-The-Wall Human Recognition”.Intelligent Environment, IEEE Global Conference on Signal and Information Processing (GlobalSIP), 2016.

22. ShijunZhai*,Ting Jiang, "Target detection and classification by measuring and processing bistatic UWB radarsignal'https://doi.org/10.1016/j.measurement.2013.08.031, Volume 47, 2014.

23. Li Cuimei, Qi Zhiliang, Jia Nan, Wu Jianhua "Human face detection algorithm via Haar cascade classifier combined with threeadditional classifiers" IEEE 13th International Conference on Electronic Measurement \& Instruments 2007.

\section{Author ProfiLe}

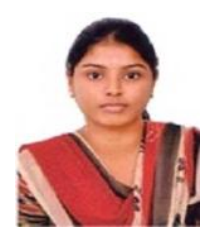

Shakthi Priyadharshini was born in thanjavur, Tamilnadu. She received her B.E, Computer Science and Engineering in Kings College of Engineering, Thanjavur. Currently, she pursues her M.Tech Computer Science in Sastra Deemed To be University, Thanjavur. Her area of interest includes Internet of Things, Image Processing, Software Engineering, Database Management 